\title{
Degradation of human cartilage by synovial fluid but not cytokines in vitro
}

\author{
Anthony P Hollander, Roger M Atkins, Deborah M Eastwood, Paul A Dieppe, \\ Christopher J Elson
}

\begin{abstract}
Synovial fluids from patients with rheumatoid arthritis and from patients with osteoarthritis degraded the matrix of living, but not killed, normal human cartilage as judged by loss of glycosaminoglycans. Normal human serum did not degrade living cartilage and neither, unexpectedly, did recombinant human cytokines.
\end{abstract}

Cartilage degradation in rheumatoid arthritis (RA) and osteoarthritis (OA) is considered to be mediated by interleukin 1 (ILl) and tumour necrosis factor, which act indirectly by stimulating chondrocytes to produce degrading enzymes. ${ }^{1-5}$ Proteolytic enzymes acting directly on the cartilage matrix might also cause degradation. ${ }^{6}$ Previous studies have shown the degradative effects of purified or recombinant cytokines on animal cartilage. ${ }^{1-5}$ Here we report the effects of arthritic synovial fluid and recombinant cytokines on the concentration of glycosaminoglycans in living and killed human cartilage in vitro.

\section{Patients and methods}

Samples of knee synovial fluid were obtained from patients with RA and OA. All patients with RA fulfilled the American Rheumatism Association criteria for classical or definite RA and were receiving a variety of non-steroidal anti-inflammatory agents and slow acting antirheumatic drugs. All patients with OA showed clear radiographic and clinical evidence of the disease. The synovial fluids were collected in sodium citrate $(3 \% \mathrm{w} / \mathrm{v})$, centrifuged, treated with hyaluronidase, and stored at $-70^{\circ} \mathrm{C}$ until Department of Pathology A P Hollander C J Elson

Department of Orthopaedic Surgery, University of Bristol, UK R M Atkins

D M Eastwood

Department of

Rheumatology,

University of Bristol, UK

P A Dieppe

Correspondence to:

Dr C J Elson,

Department of Pathology,

Medical School, University of Bristol, University Walk

Bristol BS8 1TD, UK

Accepted for publication 14 August 1990 at the time of operative fixation. Slic arthritic cartilage were obtained from patients with RA and OA undergoing total hip or knee replacement. Ten $3 \times 3 \mathrm{~mm}$ biopsy specimens cases small samples of cartilage, which could not be reconstructed into the joint surface owing to fracture comminution, were removed fem os abcapital fracture were taken at random from cartilage slices of each joint and used to determine the intrinsic glycosaminoglycan content of the cartilage.

For culture experiments biopsy specimens were cut in half and the two pieces were placed in separate wells of a 24 well plate. One of the pieces (control biopsy specimen) was cultured in $2 \mathrm{ml}$ of medium (RPMI 1640 supplemented with glutamine, penicillin streptomycin, HEPES ( $N$-2-hydroxyethylpiperazine- $N^{\prime}-2$ ethanesulphonic acid) and $5 \%$ heat inactivated normal human AB serum). The other piece (treated biopsy specimen) was cultured in $2 \mathrm{ml}$ of medium containing one of the following stimuli: $15 \%$ synovial fluid, $15 \%$ normal human serum, recombinant human ILl $\alpha$, ILl $\beta$, or tumour necrosis factor $\alpha$. Each synovial fluid, normal human serum, or cytokine was tested on five biopsy specimens alongside five paired control biopsy specimens. The cultures were incubated for 48 hours at $37^{\circ} \mathrm{C}$ in an atmosphere of humidified $5 \% \mathrm{CO}_{2} /$ air. At the end of the culture period the plates were stored at $-20^{\circ} \mathrm{C}$ until the glycosaminoglycan concentration in each biopsy specimen was determined.

Estimation of sulphated glycosaminoglycans in cartilage was carried out by the colorimetric method of Farndale $e t a l,{ }^{7}$ modified to allow use of a 96 well microtitre plate reader to measure the colour change. The loss of glycosaminoglycans from cartilage induced by each synovial fluid, normal human serum, or cytokine was estimated by the following equation:

$$
\begin{aligned}
& \text { \% GAG loss }= \\
& 100-\left(\frac{\text { mean [GAG] in } 5 \text { treated biopsy specimens }}{\text { mean [GAG] in } 5 \text { control biopsy specimens }} \times 100\right)
\end{aligned}
$$

where $\mathbf{G A G}=$ glycosaminoglycan.

The significance of differences between groups of data was tested using Student's two tailed $t$ test. A value of $\mathrm{p}<0.05$ was taken as significant.

\section{Results}

In preliminary experiments the intrinsic concentration of glycosaminoglycans was compared in arthritic and normal cartilage. The mean (SD) glycosaminoglycan concentration in normal hip and knee cartilage from 17 patients was $31 \cdot 4(4 \cdot 6) \mu \mathrm{g} / \mathrm{ml}$. The equivalent values for cartilage from nine patients with RA and 11 with OA were $8.1(7 \cdot 7) \mu \mathrm{g} / \mathrm{mg}$ and $18.5(6.7)$ $\mu \mathrm{g} / \mathrm{mg}$ respectively. The three groups were all 
$R A$ synovial fluid induced glycosaminoglycan (GAG) loss from live and killed cartilage. Biopsy specimens were taken from one normal femoral head. Each line represents one synovial fluid. ${ }^{*} p<0 \cdot 01$.

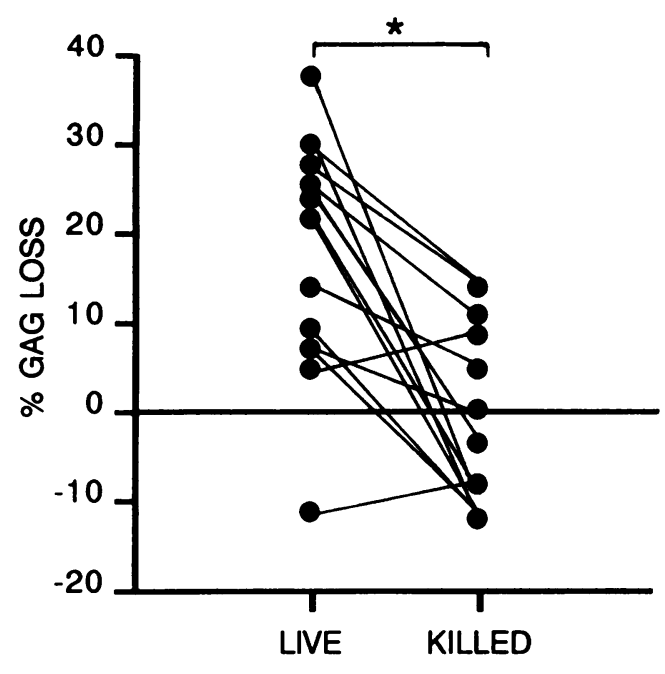

significantly different from each other $(p<0.01$ for normal $v \mathrm{RA}$ and normal $v \mathrm{OA} ; \mathrm{p}<0.05$ for RA $v$ OA).

The glycosaminoglycan content of cartilage biopsy specimens was measured after incubation for two days with or without synovial fluid or normal human serum. Rheumatoid arthritis and OA synovial fluids induced up to $38 \%$ loss of glycosaminoglycans from normal cartilage (mean (SD) for RA synovial fluid 17.6 (11.2) $\mu \mathrm{g} / \mathrm{mg} ; \mathrm{n}=27$ and for OA synovial fluid 19.8 (10.9) $\mu \mathrm{g} / \mathrm{mg} ; \mathrm{n}=5$ ), whereas normal human serum induced no more than $14 \%$ glycosaminoglycan loss (mean (SD) $4 \cdot 8 \pm(7 \cdot 1) \mu \mathrm{g} / \mathrm{mg} ; \mathrm{n}=5$ ). These differences were significant $(p<0.01$ for RA synovial fluid $v$ normal human serum and $\mathrm{p}<0.05$ for OA synovial fluid $v$ normal human serum). In subsequent experiments RA synovial fluids were found to induce a similar glycosaminoglycan loss from RA cartilage. To determine whether synovial fluid degrades cartilage directly or by stimulating chondrocytes, $15 \mathrm{RA}$ synovial fluids were tested for their ability to degrade normal cartilage which had been killed by repeated freeze thawing. As shown in the figure the RA synovial fluids degraded dead cartilage significantly less well than they degraded living cartilage $(p<0.01)$. However, $10 \mathrm{ng} / \mathrm{ml}$ of recombinant human ILl $\alpha$, ILl $\beta$, or tumour necrosis factor $\alpha$ induced little or no loss of glycosaminoglycans from normal human cartilage, even when used in combination with each other. The mean (SD) percentage glycosaminoglycan loss in six experiments was 1 (12) for ILl $\alpha,-2$ (12) for ILl $\beta, 3$ (7) for tumour necrosis factor $\alpha$, and -4 (1) for the mixture of all three cytokines. Higher and lower concentrations of the cytokines were equally ineffective (data not shown). The recombinant human IL $\alpha$ and ILI $\beta$ were both effective in stimulating proliferation of mouse thymocytes and their activity was inhibited by specific neutralising antisera (data not shown).

\section{Discussion}

Depletion of cartilage matrix, both close to and distant from the cartilage/synovium junction, is a common feature of $\mathrm{RA},{ }^{8}$ and we have found that cartilage from patients with severe arthritis has a significantly lower glycosaminoglycan concentration than normal cartilage. The results with synovial fluid reported here support the hypothesis that these differences are due to degradation of cartilage matrix by components of synovial fluid, as both RA and OA synovial fluids induced glycosaminoglycan loss from living human cartilage in vitro. Killed cartilage was not affected by RA synovial fluid, indicating that the degradation is chondrocyte dependent and probably, therefore, endogenous ILl and tumour necrosis factor in the synovial fluid play a part. 12

Surprisingly, recombinant cytokines, either on their own or in combination with each other, and at concentrations similar to those in RA synovial fluid, 910 were unable to degrade normal human cartilage in this system. These results contrast strikingly with previous studies using animal cartilage ${ }^{1-5}$ and suggest that cytokines are not enough on their own to degrade human cartilage. We suggest that the cytokines in synovial fluid can only act on human cartilage synergistically with some other component(s) of synovial fluid, such as cartilage degrading proteases. $^{6}$

This study was supported by the UK Medical Research Council (MRC). APH is in receipt of a UK MRC postgraduate studentship. Recombinant human ILl $\alpha$ was a gift from Hoffman La-Roche, New Jersey, recombinant human ILl $1 \beta$ was a gift from
Roussel, UCLAF, and recombinant human tumour necrosis Roussel, UCLAF, and recor
factor $\alpha$ a gift from Glaxo.

I Steinberg J J, Hubbard J R, Sledge C B. Chondrocytemediated breakdown of cartilage. $\mathcal{F}$ Rheumatol 1987; 14: $55-8$

2 Dingle J T. The role of cellular interactions in joint erosions. Clin Orthop 1984; 182: 24-30.

3 Henderson B, Pettipher E R. Comparison of the in vivo inflammatory activities after inter-articular injection of natural and recombinant IL-1 $\alpha$ and IL-1 $\beta$ in the rabbit. natural and recombinant $14-1 \alpha$ and

4 Gowen M, Wood D D, Ihrie E J, Meats J E, Russell R G Stimulation by human interleukin 1 of cartilage breakdown and production of collagenase and proteoglycanase by and production of collagenase and proteglycanase by human chondrocytes but not by human ost
Biochim Biophys Acta 1984; 797: 186-93. 5 Saklatvala J. Tumour necrosis factor $\alpha$ stimulates resorption and inhibits synthesi

6 Harris E D Jr, DiBona D R, Krane S M. Collagenase in human synovial fluid. $\mathcal{F}$ Clin Invest 1969; 48: 2104-13.

7 Farndale R W, Buttle D J, Barret A J. Improved quantitation and discrimination of sulphated glycosaminoglycans by use of dimethylmethylene blue. Biochim Biophys Acta 1986; 883: $173-7$.

8 Mitchell N S, Shepard N. Changes in proteoglycan and collagen in rheumatoid arthritis. F Bone foint Surg [Am] 1978; 60: 349-54.

9 di Giovine F S, Poole S, Situnayake R D, Wadhwa M, Duff G W. Absence of correlation between indices of systemic inflammation and synovial fluid interleukin 1 (alpha and beta) in rheumatic diseases. Rheumatol Int 1990; 9: 259-64. reactive tumour necrosis factor alpha and biological inhibitor(s) in synovial fluid from rheumatic patients. 1988; 1: 17-26. 